\title{
Spectroscopic biomedical imaging with the Medipix2 detector
}

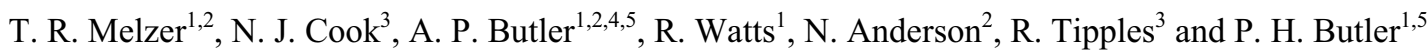 \\ ${ }^{1}$ Physics and Astronomy, University of Canterbury, Christchurch, New Zealand \\ ${ }^{2}$ Christchurch Medical School, University of Otago, Christchurch, New Zealand \\ ${ }^{3}$ Canterbury District Health Board, Christchurch, New Zealand \\ ${ }^{4}$ Electrical and Computer Engineering, University of Canterbury, Christchurch, New Zealand \\ ${ }^{5}$ European Organisation for Nuclear Research, Geneva, Switzerland
}

\begin{abstract}
This study confirms that the Medipix $2 \mathrm{x}$-ray detector enables spectroscopic bio-medical plain radiography. We show that the detector has the potential to provide new, useful information beyond the limited spectroscopic information of modern dual-energy computed tomography (CT) scanners. Full spectroscopic 3D-imaging is likely to be the next major technological advance in computed tomography, moving the modality towards molecular imaging applications. This paper focuses on the enabling technology which allows spectroscopic data collection and why this information is useful. In this preliminary study we acquired the first spectroscopic images of human tissue and other biological samples obtained using the Medipix2 detector. The images presented here include the clear resolution of the $1.4 \mathrm{~mm}$ long distal phalanx of a 20 -week-old miscarried foetus, showing clear energy-dependent variations. The opportunities for further research using the forthcoming Medipix3 detector are discussed and a prototype spectroscopic CT scanner (MARS, Medipix All Resolution System) is briefly described.
\end{abstract}

Key words Medipix, spectroscopic x-ray imaging, single photon counting

\section{Introduction}

\section{Dual-energy and spectroscopic CT}

Until recently, medical x-ray imaging has lacked the ability to discriminate between different photon energies ${ }^{1}$. With this study, our group becomes the first to acquire 2D spectroscopic images of human tissue using the Medipix2 detector ${ }^{2}$. This is an important first step in enabling the development of a practical spectroscopic Computed Tomography (CT) system.

CT has become an indispensable component of virtually every radiology department ${ }^{1,3}$; improvements in speed and resolution have seen it emerge as the workhorse for many radiological studies. Despite its usefulness, current CT detector systems waste much of the information carried by incident photons. Most CT advancements have been to improve the use of absorption information and to reject scattering information. However diffraction,

Corresponding author: Anthony P. Butler, Physics and Astronomy, University of Canterbury, Christchurch, New Zealand Tel: (+64) 3 3640913, Fax: (+64) 33640620

Email: anthony@butler.co.nz

Received: 29 September 2008; Accepted: 8 December 2008

Copyright (C) 2008 ACPSEM interference, and energy information have remained undetectable and untapped except in the recently developed dual energy x-ray CT systems ${ }^{1}$.

Of all the areas of possible improvement for radiation detectors, energy discrimination of incident x-ray photons is the most promising ${ }^{1,4-6}$. The first steps along the path toward spectroscopic CT have already been taken by dualenergy CT. This technology has demonstrated that, even with the relatively simple information yielded by overlapping spectra, there are significant improvements to be made to image quality and content.

Digital subtraction of dual-energy CT images uses differences in attenuation to identify different tissues or contrast agents ${ }^{3,7-8}$. The method requires two scans at different energies, which are digitally subtracted to exploit different tissue's energy-dependent absorptions. The resulting image information can be used to diagnose a wide variety of abnormalities including the difference between benign and malignant growths ${ }^{9}$, calcifications including microcalcifications in mammography, calcified pulmonary nodules, and calcified lymph nodes not visible in standard projection radiographs 8,10 . Dual-energy subtraction also assists in the identification of mediastinal masses, the quantification of vascular disease, recognition of bone, pleural, and chest wall abnormalities, and the localization of devices such as stents and catheters which are often difficult to distinguish using plain projection radiographs ${ }^{11}$. Dual-energy CT systems have been developed using X-rays 
from a single source (fast $\mathrm{kV}$ switching) as well as a dualsource $^{1}$ (X-ray tubes at $90^{\circ}$ ). They are useful for the above reasons but do exhibit time lag and count rate limitations; they are also limited to two energies with overlapping x-ray spectra.

Spectroscopic CT would significantly enhance this already useful technology ${ }^{4-5}$ by dividing a single, broad Xray spectrum into definable and separate energy bands. Allocating the energy of each detected photon to one of these bands will increase tissue discrimination and alleviate many current imaging problems with consequent improvement to imaging practices ${ }^{12}$. Others have acknowledged $^{1,6}$ the expected benefits of energy sensitive $\mathrm{CT}$ and are actively pursuing different methods of attaining this goal; to date their published efforts have not resulted in a practical detector technology.

It is difficult to predict the exact consequences that spectroscopic capabilities will have on x-ray imaging, but numerous fields within $\mathrm{CT}$ are poised to make considerable advancement with this technology ${ }^{13-15}$. For example, beam hardening causes characteristic artefacts in reconstructed images when areas of low attenuation are falsely computed around areas of high attenuating materials ${ }^{4-5,12}$. Materials such as metallic implants distort the area just around the implant, which often corresponds to the region of clinical interest ${ }^{4,12}$. Current correction methods (filtering or software algorithms) are imperfect, but energy information may completely eliminate these artefacts ${ }^{1,5}$ by measuring the transmitting spectrum then compensating for the hardening effect.

A spectroscopic CT scanner would also reduce the dose required to obtain contrast-dependent images; images from existing multi-phase contrast protocols could be reconstructed from a single data acquisition. With energy resolving detectors, the contrast agent could be selected and viewed or ignored, facilitating superior identification of tumours or plaques with decreased dose $\mathrm{e}^{14}$. With a single acquisition, patient movement becomes a non-issue and productivity increases. Less contrast agent is required, providing benefits for the elderly and patients with renal failure or diabetes. These factors translate to safer, as well as cheaper, CT imaging practices ${ }^{4,12,15}$.

Spectroscopic information will also yield better intrinsic tissue contrast ${ }^{4-5}$. Different tissues have different, energy-dependent attenuation properties depending on their atomic makeup. Current dual-energy techniques exploit these differences to identify specific tissues; full spectroscopic imaging would enhance this ability ${ }^{3-5,9,16-17}$.

\section{Dual-energy Medipix2 imaging}

In this preliminary study, we focus on demonstrating the practical application of the Medipix2 detector to plain, dual-energy imaging of bio-medical samples. Future work will apply the principles developed here to $3 \mathrm{D}$ imaging where the removal of overlying tissues markedly increases the power of the technique.

CERN (Conseil Européen pour la Recherche Nucléaire) designed and developed the Medipix2 detector ${ }^{18}$. The photon counting pixel detector uses a semiconductor sensor layer to directly convert $\mathrm{x}$-ray photons into electron-hole pairs. Under the influence of a bias voltage, the electrons or holes are collected by a separate electronics layer where the signal processing occurs. The charge pulses created by the electrons or holes are individually analysed and counted. The pixelated sensor and electronics layers allow for real time signal processing and in-pixel digitising ${ }^{19}$. These features of Medipix2 allow for good spatial and temporal resolution, as well as very low noise $\mathrm{e}^{2,20}$.

One of the most exciting capabilities of the Medipix2 chip is its ability to discriminate among different photon energies, as demonstrated by Tlustos et al ${ }^{20}$. The energy window feature of the Medipix2 chip can select specific photon energies from the range of energies produced by a broad bremsstrahlung source. The detector has two pulse height discriminators, which can be configured to capture images in a specific energy band. In this manner, two images are acquired from the same source, one at high energy and the other at low energy which, when subtracted, reveal absorption differences. The planned Medipix3 detector described by Ballabriga et $a l^{18}$ has multiple energy windows facilitating simultaneous capture of images in several energy bands giving single acquisition, full spectroscopic imaging.

The technical features of the Medipix2 detector-its energy discrimination capabilities, superior spatial resolution, low noise, and fast readout - make the detector a good candidate for a prototype spectroscopic CT system $^{20,21}$. Such a system has been designed and built by our group. It has been named the Medipix All Resolution System (MARS) and we will present full spectroscopic, 3D images from this system in future publications.

\section{Materials and methods}

The work for this paper was carried out on a decommissioned CTG Senographe 600T (General Electric Medical Systems, WI, USA) and a Siemens 3000 Nova Mammomat (Siemens Medical Solutions, Ltd., Erlangen, Germany) machine still in clinical use. This project was approved by the local Christchurch School of Medicine Ethics Committee (URB/07/02/001).

Medipix2 (Figure 1) is a complementary metal-oxide semiconductor with single photon counting capabilities ${ }^{2}$. The detection system contains a matrix of $256 \times 256$ pixels. Each $55 \times 55 \mu^{2}$ pixel contains a low noise preamplifier and two pulse height discriminators. The readout chip is bump bonded to a $300 \mu \mathrm{m}$ silicon semi-conducting sensor layer for direct charge conversion of incoming photons. This type of hybrid chip separates the detector layer from the electronics layer allowing the use of different detector materials. The chip is read out serially by the MUROS2 (Medipix2 re-Usable Read-Out System version 2) electronic interface controlled by the software package Pixelman (Czech Technical University, Prague) ${ }^{22}$.

One of the issues with hybrid detectors is the charge sharing which occurs when multiple pixels register hits from a single incident photon interaction with the semiconducting layer ${ }^{2,22-23}$. The charge cloud produced by 


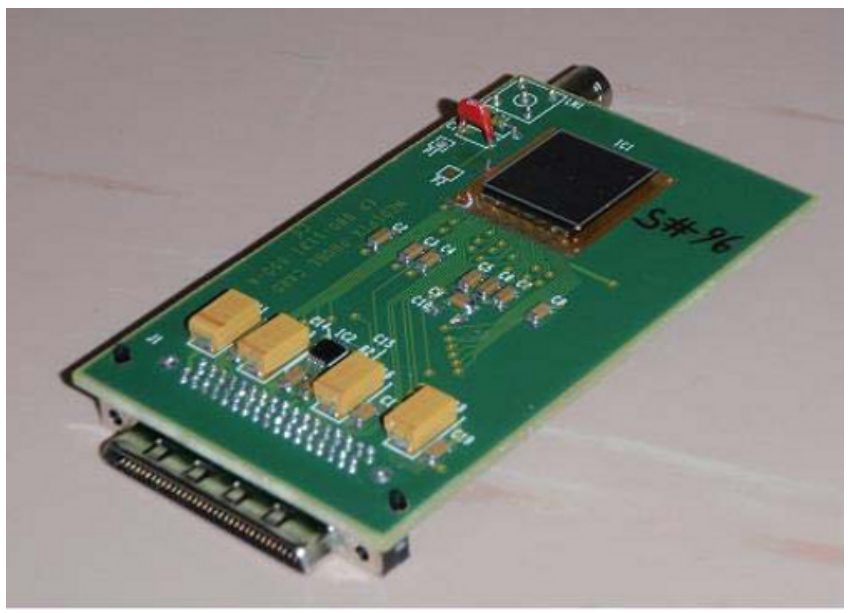

Figure 1. Photo of the Medipix2 detector with the semiconductor sensor layer bump bonded to the read-out chip.

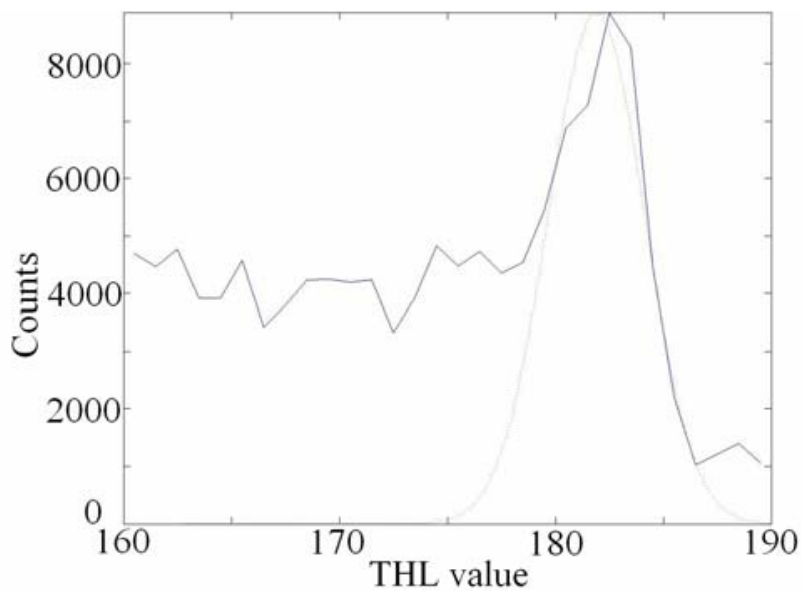

Figure 2. I-125 Spectrum: Counts vs. THL. The spectrum of an I125 source with charge sharing effects present (non-zero counts outside the peak). The peak (27.5keV) corresponds to a THL value of approximately 181.

the ncident -ray hoton spreads out as it travels through the semiconductor under the influence of the bias voltage. Upon reaching the electronics layer, the charge cloud an cover many pixels, each of which records a hit of some fraction of the original photon energy. This sharing of charge degrades the system's energy and spatial resolution, especially at lower energies ${ }^{2,22}$. Increasing the bias voltage reduces this effect by decreasing the time for charge to spread among adjacent pixels but cannot eliminate it.

\section{Chip calibration}

The threshold equalisation process corrects for inhomogeneities in the pixel response due to variations in the electronics layer, particularly the variation of preamplifier gain ${ }^{19}$. Every pixel contains a 3-bit threshold adjustment that can be manipulated to equalize that pixel's response. We created the low threshold equalisation distribution by following a method outlined in Bisgoni et $a l^{24}$ and Tlustos et $a l^{20}$ which, when applied across the detector array, compensates for electronic inconsistencies and variation, giving a flatter response.
Detector function is controlled by a number of Digital to Analogue Converters (DACs). The detector functions in single energy threshold mode if the THH (High Threshold discriminator) DAC is set lower than the THL (Low Threshold discriminator) DAC. In this configuration all photons with energies greater than THL are recorded. If the THH is set above the THL, the detector will perform in energy window mode and will only record photons with energies between the low and high thresholds.

The high threshold equalisation distribution is calculated after the determination of a suitable low threshold distribution. The low threshold is calculated using the noise floor of the chip as a reference but the high threshold is calculated using the crossing point of the low and high threshold distributions ${ }^{20}$. This means that the high threshold distribution can never be as accurate as the low since it will be broadened by the low energy distribution. The images discussed later in this paper were therefore acquired using the detector in the single, low energy threshold mode.

\section{Photon energy scale calibration}

Imaging an Iodine-125 seed (peak emission $27.5 \mathrm{keV}$ ) permitted the calibration of the threshold DACs (THL and $\mathrm{THH}$ ) to $\mathrm{x}$-ray photon energies. This makes the investigation of known energy windows possible. Scanning in one DAC increments from the noise floor of THL 155 (THH 0) to THL 200, the detector recorded the cumulative signal deposited by the seed. Then, following the method outlined in Tlustos et $a l^{20}$, the count rates from individual pixels were differentiated with respect to energy and normalized, yielding the iodine spectrum (Figure 2). THL $\sim 181$ therefore corresponds to $27.5 \mathrm{keV}$ and the noise floor usually falls between $2-5 \mathrm{keV}^{19}$. Bisgoni et a ${ }^{24}$ demonstrated a linear relationship between DAC values and photon energy; therefore, an approximate energy scale can be assigned to the THL values using the peak energy of iodine and the assumed energy of the noise floor. One unit on the DAC scale corresponds to a photon energy of between 1.02 and $1.16 \mathrm{keV}$.

\section{Beam hardening}

The silicon detector pixels exhibit an energy-dependant absorption efficiency which leads to increased image noise due to beam hardening. The Pixelman software has a routine to compensate for this effect by obtaining flat-field images for varying degrees of beam hardening and plotting the response of each pixel ${ }^{25}$. The efficiency of each pixel can therefore be decoupled from photon energy and the noise due to beam hardening is reduced. This calibration corrects for the variation in each pixel's response to different energies but retains tissue dependent absorption information $^{22}$.

\section{Biological sample image acquisition}

As an initial step, we imaged sections of chicken wing to confirm that the detector and calibrations were working properly. The CTG Senographe 600T was used to capture single threshold images at THL 155 (approx 2.5keV) and 165 (approx 13.4keV) using a tube voltage of $36 \mathrm{kVp}$ 


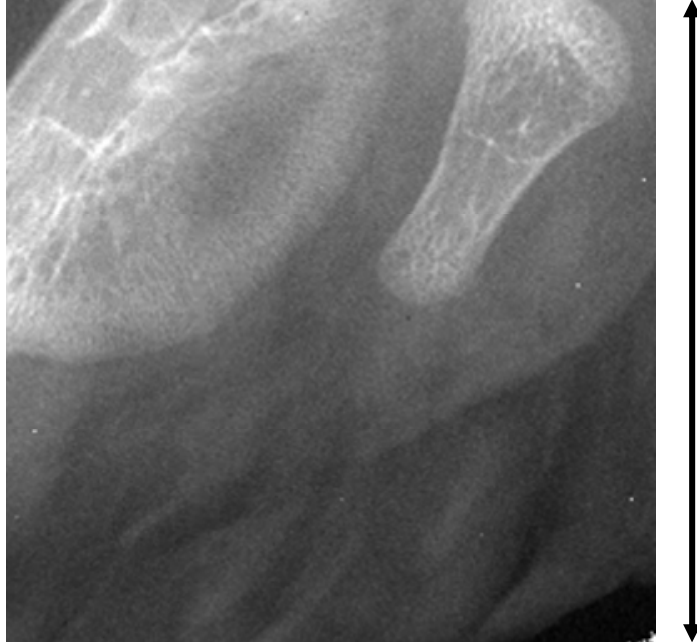

(a)

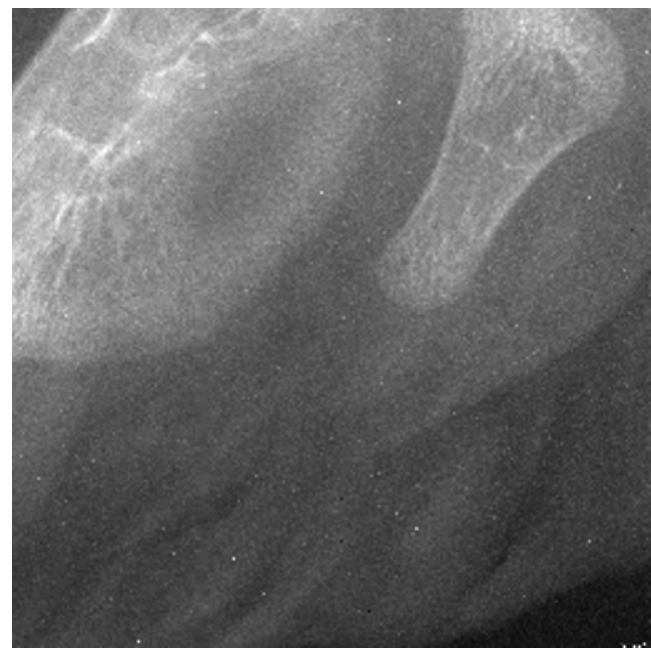

(b)

(Figure $3 \mathrm{a}, 3 \mathrm{~b}$ ). The exposure of each acquisition was adjusted to maintain constant detected counts. The specific beam hardening calibration appropriate to each THL value was applied to each image and dead pixel columns were linearly interpolated. We employed Matlab (The Mathworks, Massachusetts, USA) to digitally subtract the single threshold images (Figure 3c). The greyscale window and level values were adjusted to display maximum detail in each image.

We also acquired images of the left hand of a 20week-old miscarried fetus (Siemens 3000 Nova Mammomat at $35 \mathrm{kVp}$ ). Single threshold images were acquired for the same THL values (155 and 165) and the exposure times were adjusted as before (Figure 4a, 4b). The same process of beam hardening compensation and digital subtraction was performed (Figure 4c).

\section{Results}

Projection and dual-energy subtracted images demonstrate clinical quality over the $14 \mathrm{~mm} \times 14 \mathrm{~mm}$ field of view. Figure 3 shows the single threshold projection images of the chicken wing. Taken at the lowest threshold
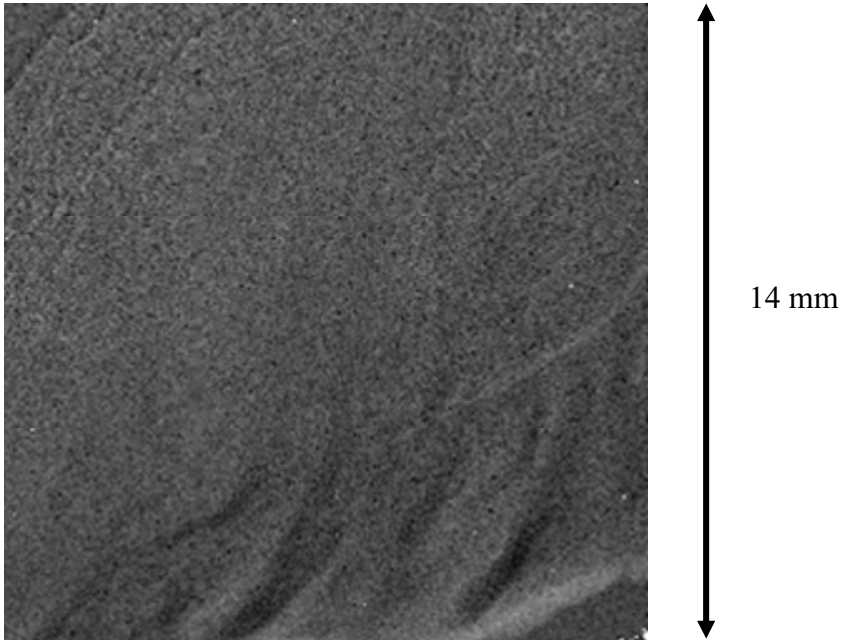

(c)

Figure 3. Images of the tip of a chicken wing; all the images have an area of $14 \times 14 \mathrm{~mm}^{2}$. (a) The image was acquired at THL $155(E>2.5 \mathrm{keV})$, just above the noise floor. (b) The same image taken at THL $165(E>13.4 \mathrm{keV})$. Soft tissue detail is superior in (a) due to the contribution of the lower energy photons. These low energy photons are rejected at the higher THL in (b). Bony detail is preserved in both images. (c) Dualenergy subtraction image of a chicken wing. THL $165-155$ : $(E>13.4 \mathrm{keV}-E>2.5 \mathrm{keV})$. The subtraction highlights soft tissue contrast while removing bone information. In the clinical environment, this technique helps differentiate between benign and malignant tissues. above the noise floor (THL=155), Figure 3 a exhibits both bony detail as well as soft tissue information. Figure $3 b$ rejects these lower energy photons and subsequently displays slightly less soft tissue contrast.

Digitally subtracting two images obtained at different single thresholds produces a dual-energy subtraction image. Between the two images, the absorption of bone varies little, while soft tissue attenuation changes significantly. Upon subtraction, the unchanging bone is removed and the subtle variations in soft tissue absorption are obvious upon visual inspection (Figure $3 \mathrm{c}$ ).

Figure 4 shows the left hand of a 20-week-old miscarried fetus. The Medipix 2 system clearly images the $1.4 \mathrm{~mm}$ long distal phalanx in the upper left hand corner of the image. Figures $4 \mathrm{a}$ and $4 \mathrm{~b}$ are singlethreshold projection images acquired at just above the noise floor (THL 155) and at THL 165 respectively. The images exhibit good spatial resolution, high contrast, and low noise. The digitally subtracted image in Figure $4 \mathrm{c}$ demonstrates the ability to discriminate among different photon energies and shows the absorption variation due to different stages of calcification of the bones. 


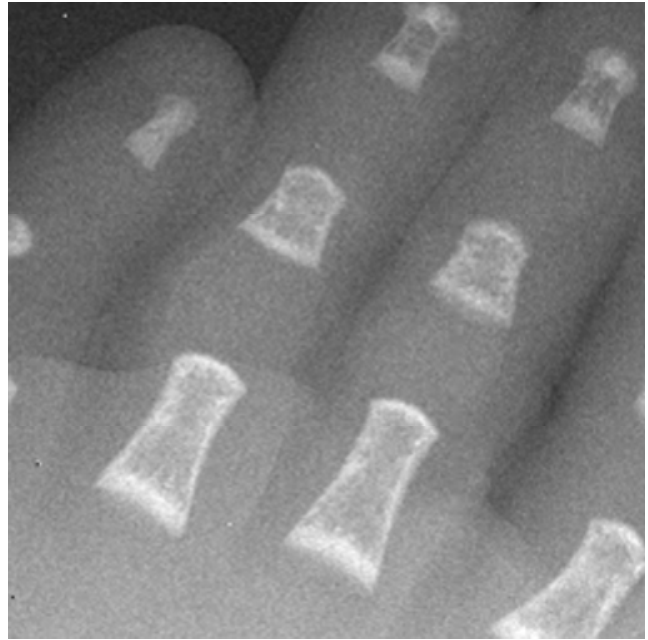

(a)

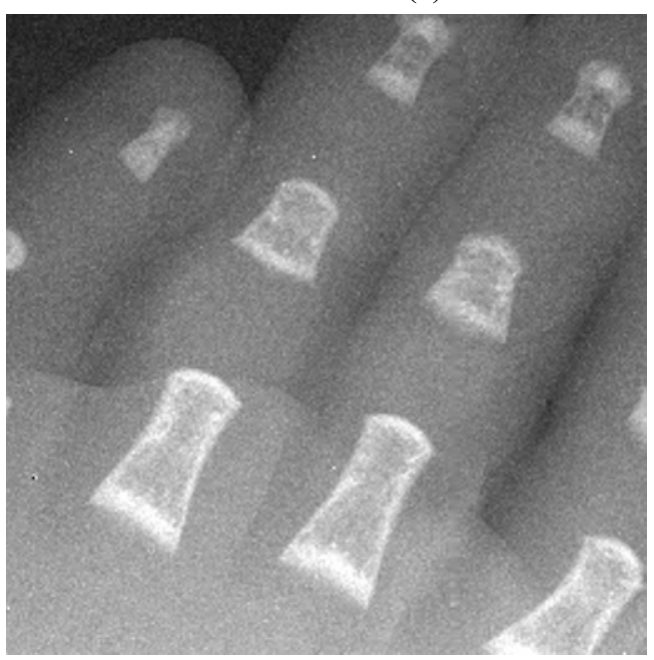

(b)

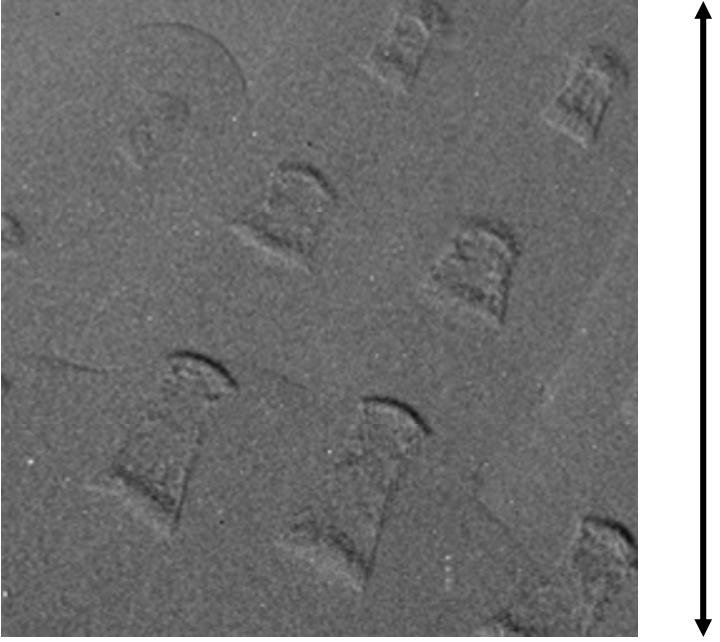

(c)

Figure 4. Fingers of a fetal left hand. (a) THL 155: (E $>2.5 \mathrm{keV})$ The images display very good spatial resolution. The small, developing bones and soft tissue of the hand can be seen quite clearly. The small bone in the upper left hand corner measures less than 1.4mm in length. (b) THL 165: (E>13.4keV) Eliminating low energy photons demonstrates slight differences in absorption that can be seen in the soft tissue and at the edges of bone. (c) THL 165 - 155: The subtraction image emphasises the different tissues present. The added amount of material at the edge of the growing bone causes a sharp increase in contrast and can be seen clearly in the image. The soft tissue of the fingers, in contrast, is uniform. The sharp change in contrast highlighted by the subtraction can be very useful in the identification of microcalcifications in mammography.

\section{Discussion}

The clinical images produced in this study exhibit two exciting features of the Medipix2 detector. The detector's ability to set energy thresholds allows for energy discrimination and spectroscopic imaging through dualenergy digital subtraction. Secondly, the radiological images demonstrate high quality spatial resolution, low noise, and good contrast.

We obtained both high and low energy images using the adjustable energy threshold at a constant tube $\mathrm{kVp}$. Current technologies require a dual-energy source with two distinct tube $\mathrm{kVp}$ 's, or a sandwich setup with two separately filtered detectors to produce images required for subtraction. Medipix2 uses a constant $\mathrm{kVp}$ bremsstrahlung source and selects the energy ranges digitally.

The $27.5 \mathrm{keV}$ gamma emission peak of Iodine-125 approximately corresponds to THL value of 181. Another one or more precisely defined reference points (e.g. gamma emissions) would facilitate investigation of specific photon energies. In the future, k-emission lines from irradiated metal foils will be used for such a calibration.
The silicon semiconductor layer has very limited absorption efficiency at diagnostic X-ray energies. Improved production processes will make available higher $\mathrm{z}$ detector materials such as GaAs and CdTe, increasing the functionality of Medipix by expanding its useful range to higher, diagnostic energies $(>100 \mathrm{keV})^{23,26}$.

The small size of the chip $\left(14 \times 14 \mathrm{~mm}^{2}\right)$ limits its use in everyday clinical application. For general plain radiologic imaging, the chip would need to cover a much larger area. The most promising application at present is to include a line of detectors in a computed tomography system. This technique eliminates the need for a large detector and provides the added benefit of 3-D data sets. Exploiting this solution, our group has designed and built a desktop, spectroscopic CT scanner (MARS) to further explore the capabilities of Medipix2, as well as the forthcoming Medipix3. The next generation chip, Medipix3, will have 8 independent threshold values which will permit full spectroscopic imaging with a single exposure. Additionally, neighbouring pixels will communicate with one another on an event by event basis, to reduce the effects of charge sharing. These advances will create true, spectroscopic images with improved energy and 
spatial resolution ${ }^{18}$. With a move to $3-\mathrm{D}$ spectroscopic reconstructions, the mass attenuation curve of each particular structure within the body could be individually identified, decreasing artefacts and improving diagnostic power ${ }^{4}$. For these reasons, spectroscopic CT (MARS) is the logical next step in the application of Medipix to clinical imaging applications.

\section{Conclusion}

This study achieved two principle objectives: the Medipix2 detector extracted energy information from the $\mathrm{x}$-ray beam and produced clinical quality radiological images. This paper outlines the specific methods used in applying Medipix technology to medical imaging. The application of dual-energy subtraction techniques to this new technology produced images that form the foundation of true spectroscopic $x$-ray imaging by demonstrating energy selectivity. Furthermore, the single threshold projection images exhibit the ability of the detector to capture $\mathrm{x}$-ray images of acceptable standard. We therefore believe that biomedical spectroscopic CT is a feasible and promising direction in which to progress with Medipix to deliver routinely available energy selectivity.

\section{Acknowledgements}

Many thanks to the Department of Physics and Astronomy at the University of Canterbury, the Christchurch School of Medicine of the University of Otago, the Departments of Radiology, and Medical Physics and Bioengineering at Christchurch Hospital. We would like to thank the Royal Australian and New Zealand College of Radiologists for their financial support.

\section{References}

1. Wang, G., Yu, H. and De, Man B., An outlook on x-ray $C T$ research and development, Med. Phys., 35(3): 1051-64, 2008.

2. Llopart, X., Campbell, M., Dinapoli, R., San Segundo, D. and Pernigotti, E., Medipix2: a 64-k pixel readout chip with 55- $\mu \mathrm{m}$ square elements working in single photon counting mode, IEEE Trans. Nucl. Sci., 49(5): 2279-83, 2002.

3. Bushberg, J. T., Seibert, J. A., Leidholdt, Jr. E. M. and Boone, J. M., The Essential Physics of Medical Imaging, 2nd ed., Lippincott Williams and Wilkins, Philadelphia, 2001.

4. Butler, A. P. H., Anderson, N. G., Tipples, R., Cook, N., Watts, R., Meyer, J., Bell, A. J., Melzer, T. R., Butler, P. H., Bio-medical $x$-ray imaging with spectroscopic pixel detectors, Nucl. Intrum. Meth. Phys. Res. A, 591(1): 141-6, 2008.

5. Roessl, E. and Proksa, R., K-edge imaging in $x$-ray computed tomography using multi-bin photon counting detectors, Phys. Med. Biol., 52(15): 4679-96, 2007.

6. Kraft, E., Fischer, P., Karagounis, M., Koch, M., Krueger, H., Peric, I., Wermes, N., Herrmann, C., Nascetti, A., Overdick, M. and Ruetten, W., Counting and integrating readout for direct conversion x-ray imaging: Concept, realization and first prototype measurements, IEEE Trans. Nucl. Sci., 54(2): 383-90, 2007.
7. Hoheisel, M., Review of medical imaging with emphasis on $x$ ray detectors, Nucl. Instrum. Meth. Phys. Res. A, 563(1): 21524, 2006

8. Kuhlman, J. E., Collins, J., Brooks, G. N., Yandow, D. R. and Broderick, L. S., Dual-energy subtraction chest radiography: what to look for beyond calcified nodules, Radiographics, 26(1): 79-92, 2006.

9. Johns P. C. and Yaffe, M. J., X-ray characterisation of normal and neoplastic breast tissues, Phys. Med. Biol., 32(6): 675-95, 1987.

10. Brettle, D. S. and Cowen A. R., Dual-energy digital mammography utilizing stimulated phosphor computed radiography, Phys. Med. Biol., 39(11): 1989-2004, 1994.

11. Fischbach, F., Freund, T., Rottgen, R., Engert, U., Felix, R. and Ricke, J., Dual-energy chest radiography with a flat-panel digital detector: revealing calcified chest abnormalities, Am. J. Roentgenol., 181(6): 1519-24, 2003.

12. Schoepf, U. J., Zwerner, P. L., Savino, G., Herzog, C., Kerl, J. M. and Costello, P., Coronary CT angiography, Radiology, 244(1): 48-63, 2007

13. Firsching, M., Giersch, J., Niederlohner, D. and Anton, G., $A$ method for stoichiometric material reconstruction with spectroscopic $x$-ray pixel detectors, IEEE Nucl. Sci. Symp. Conf. Record, 7: 4116-9, 2004.

14. Firsching, M., Niederlohner, D., Michel, T. and Anton, G., Quantitative material reconstruction in CT with spectroscopic $x$-ray pixel detectors: a simulation study, IEEE Nucl. Sci. Symp. Conf. Record, 4: 2257-9, 2006.

15. Maki, D. D., Birnbaum, B. A., Chakraborty, D. P., Jacobs, J. E., Carvalho, B. M. and Herman, G. T., Renal cyst pseudoenhancement: beam hardening effects on CT numbers, Radiology, 213(2): 468-72, 1999.

16. Asaga, T., Chiyasu, S., Mastuda, S., Mastuura, H., Kato, H., Ishida, M. and Komaki, T., Breast imaging: dual-energy projection radiography with digital radiography, Radiology, 164(3): 869-70, 1987.

17. Lemacks, M. R., Kappadath, S. C., Shaw, C. C., Liu, X. and Whitman, G. J., A dual-energy subtraction technique for microcalcification imaging in digital mammography: A signal-to-noise analysis, Med. Phys., 29(8): 1739-51, 2002.

18. Ballabriga, R., Campbell, M., Heijne, E. H. M., Llopart, X. and Tlustos, L., The Medipix3 prototype, a pixel readout chip working in single photon counting mode with improved spectrometric performance, IEEE Trans. Nucl. Sci., 54(5): 1824-29, 2007.

19. Tlustos, L., Performance and limitations of high granularity single photon processing $x$-ray imaging detectors, $\mathrm{PhD}$ Thesis Technischen Universitat Wien/CERN, 2005.

20. Tlustos, L., Ballabriga, R., Campbell, M., Heijne, E., Kincade, $\mathrm{K}$., Llopart, X. and Stejskal P., Imaging properties of the Medipix2 system exploiting single and dual energy thresholds, IEEE Trans. Nucl. Sci., 53(1): 367-72, 2006.

21. Bertuccio, G., Prospect for energy resolving $x$-ray imaging with compound semiconductor pixel detectors, Nucl. Instrum. Meth. Phys. Res. A, 546(1): 232-41, 2005.

22. Holy T., Jakubek J., Pospisil S., Uher J., Vavrik D. and Vykydal V., Data acquisition and processing software package for Medipix-2, Nucl. Intrum. Meth. Phys. Res. A, 563(1): 254-8, 2006.

23. Pellegrini, G., Maiorino, M., Blanchot, G., Chmeissani, M., Garcia, J., Lozano, M., Martinez, R., Puigdengoles, C. and Ullan, M., Direct charge sharing observation in singlephoton-counting pixel detector, Nucl. Intrum. Meth. Phys. Res. A, 573(1-2): 137-40, 2007.

24. Bisogni, M. G., Delogu, P., Fantacci, M. E., Mettivier, G., 
Montesi, M. C., Novelli, M., Quattrocchi, M., Rosso, V., Russo, P. and Stefanini, A., A Medipix2-based imaging system for digital mammography with silicon pixel detectors, IEEE Trans. Nucl. Sci., 51(6): 3081-85, 2004.

25. Jakubek, J., Data processing and image reconstruction methods for pixel detectors, Nucl. Intrum. Meth. Phys. Res. A,
576(1): 223-34, 2007

26. Autiero, M., Celentano, L., Cozzolino, R., Laccetti, P., Marotta, M., Mettivier, G., Montesi, M. C., Riccio, P., Roberti, G. and Russo, P., Experimental study on in vivo optical and radionuclide imaging in small animals, IEEE Trans. Nucl. Sci., 52(1): 205-9, 2005. 